\title{
Thrombopoietin amplifies ADP-induced HSP27 phosphorylation in human platelets: Importance of pre-treatment
}

\author{
NGUYEN THE CUONG $^{1,2}$, TOMOAKI DOI ${ }^{1}$, RIE MATSUSHIMA-NISHIWAKI ${ }^{2}$, SHIGERU AKAMATSU ${ }^{3}$, \\ GEN KUROYANAGI $^{2,4}$, AKIRA KONDO ${ }^{2,4}$, JUN MIZUTANI ${ }^{4}$, IKUO WADA $^{4}$, TAKANOBU OTSUKA ${ }^{4}$, \\ HARUHIKO TOKUDA $^{2,5}$, OSAMU KOZAWA ${ }^{2}$ and SHINJI OGURA ${ }^{1}$
}

\begin{abstract}
Departments of ${ }^{1}$ Emergency and Disaster Medicine and ${ }^{2}$ Pharmacology, Gifu University Graduate School of Medicine, Gifu 501-1194; ${ }^{3}$ Department of Anesthesiology and Critical Care Medicine, Matsunami General Hospital, Gifu 501-6062;

${ }^{4}$ Department of Orthopedic Surgery, Nagoya City University Graduate School of Medical Sciences, Nagoya 467-8601;

${ }^{5}$ Department of Clinical Laboratory, National Center for Geriatrics and Gerontology, Obu, Aichi 474-8511, Japan
\end{abstract}

Received February 6, 2013; Accepted April 3, 2013

DOI: $10.3892 /$ ijmm.2013.1345

\begin{abstract}
It has been shown that thrombopoietin (TPO) amplifies agonist-induced platelet activation. However, the precise mechanism of action of TPO has not yet been fully elucidated. We have previously reported that the adenosine diphosphate (ADP)-induced phosphorylation of heat shock protein 27 (HSP27) via the p38 mitogen-activated protein (MAP) kinase pathway correlates with the ADP-induced platelet-derived growth factor (PDGF)-AB secretion and the release of soluble CD40 ligand (sCD40L) from human platelets. In the present study, we investigated the effects of TPO on platelet activation induced by ADP. We examined the effects of TPO on ADP-induced platelet activation under different treatments: TPO was administered 15 min prior to stimulation with ADP (pre-treatment); TPO and ADP were simultaneously administered (simultaneous treatment); and TPO was administered 2 min following stimulation with ADP (post-treatment). TPO, which alone had no effect on platelet aggregation, synergistically enhanced the ADP $(1 \mathrm{mM})$-induced platelet aggregation only when it was administered prior to stimulation with ADP. Pre-treatment with TPO significantly increased the secretion of PDGF-AB and the release of SCD40L, and markedly enhanced the ADP-induced phosphorylation of p38 MAP kinase and HSP27 in the platelets. However, simultaneous treatment with TPO or TPO post-treatment failed to affect the ADP-induced platelet aggregation, the secretion of PDGF-AB, the release of sCD40L and the phosphorylation p38 MAP kinase or HSP27. These results strongly suggest that pre-treatment with TPO
\end{abstract}

Correspondence to: Dr Tomoaki Doi, Department of Emergency and Disaster Medicine, Gifu University Graduate School of Medicine, Yanagido 1-1, Gifu 501-1194, Japan

E-mail: tomo-you-kei@mbr.nifty.com

Key words: thrombopoietin, adenosine diphosphate, p38 mitogenactivated protein kinase, heat shock protein 27 , platelet-derived growth factor-AB, soluble CD40 ligand significantly amplifies ADP-induced HSP27 phosphorylation via the $\mathrm{p} 38$ MAP kinase pathway in human platelets.

\section{Introduction}

Platelet adhesion and aggregation are the first steps in thrombus formation at the injured vascular site, and play a crucial role in hemostasis. Platelets are activated by various stimuli, resulting in shape change, adhesion, aggregation and subsequently, thrombus formation. Thrombus formation is associated with the release of granule contents, such as platelet-derived growth factor (PDGF)-AB and serotonin, as well as the release of inflammatory substances, such as soluble CD40 ligand (sCD40L). These secreted and generated mediators trigger a positive feedback mechanism that potentiates platelet activation $(1,2)$.

Adenosine diphosphate (ADP), which is released from damaged cells and secreted from platelet-dense granules, contributes to the positive feedback mechanism for platelet activation by acting through $\mathrm{P} 2$ receptors on the platelet surface (1). It is recognized that ADP is an essential co-factor for the activation of platelets by other platelet agonists; however, ADP is a weak agonist for platelets compared to thrombin or collagen (1). ADP induces shape change and platelet aggregation through $\mathrm{P} 2 \mathrm{Y} 1$ and $\mathrm{P} 2 \mathrm{Y} 12$ receptors. It has been reported that $\mathrm{P} 2 \mathrm{Y} 1$ or $\mathrm{P} 2 \mathrm{Y} 12$ receptors stimulated by ADP induce the activation of p38 mitogen-activated protein (MAP) kinase and p44/p42 MAP kinase among the MAP kinase superfamily (1,3-5).

Heat shock proteins (HSPs) are expressed in a variety of cells in response to various types of biological stress, such as heat stress and chemical stress (6). HSP27 belongs to the low molecular weight HSP family (HSPB) with a molecular mass ranging from 10 to $30 \mathrm{kDa}$ (6). It is generally known that HSP27 activity is regulated by post-translational modifications, such as phosphorylation (6). HSP27 is promptly phosphorylated in response to various types of stress, as well as following exposure to cytokines and mitogens, and changes from an aggregated to a dissociated form (6). Human HSP27 is phosphorylated at three serine residues (Ser-15, Ser-78 and Ser-82). It is recognized that the phosphorylation of HSP27 is catalyzed by members of 
the MAP kinase superfamily, such as p38 MAP kinase (6). It has been shown that ADP induces HSP27 phosphorylation in human platelets (7). We have previously demonstrated that the ADP-induced phosphorylation of HSP27 via the p44/p42 MAP kinase and p38 MAP kinase pathway correlates with PDGF-AB secretion and the release of SCD40L from human platelets (8).

It is well known that thrombopoietin (TPO), which is recognized as a $\mathrm{Mpl}$ ligand or a megakaryocyte growth and differentiation factor, is a pivotal physiological regulator of megakaryocytopoiesis and platelet production (9). TPO interacts with its receptor, c-Mpl, resulting in the activation of a variety of signal transduction pathways (10-15). It has been reported that TPO alone fails to induce platelet aggregation, but potentiates platelet activation stimulated by numerous platelet agonists (12,16-18). However, the exact mechanism behind the amplification of platelets by agonists and TPO has not yet been fully elucidated. In the present study, we investigated the effect of TPO on ADP-induced human platelet activation. We demonstrate that pre-treatment with TPO amplifies ADP-induced HSP27 phosphorylation via the p38 MAP kinase pathway in human platelets.

\section{Materials and methods}

Materials. ADP was purchased from Sigma-Aldrich (St. Louis, MO, USA). Recombinant human TPO was purchased from R\&D Systems, Inc. (Minneapolis, MN, USA). p38 MAP kinase antibodies and phospho-p38 MAP kinase antibodies were obtained from Cell Signaling, Inc. (Beverly, MA, USA). HSP27 antibodies, phospho-HSP27 (Ser-15) antibodies and phosphoHSP27 (Ser-78) antibodies were from Stressgen Biotechnologies (Victoria, BC, Canada). Phospho-HSP27 (Ser-82) antibodies were from Biomol Research Laboratories, (Plymouth Meeting, PA, USA). GAPDH antibodies were purchased from Santa Cruz Biotechnology, Inc. (Santa Cruz, CA, USA). The ECL western blotting detection system was purchased from GE Healthcare (Buckinghamshire, UK). Other materials and chemicals were obtained from commercial sources.

Preparation of platelets. Human blood was donated from healthy volunteers and collected into a 1/10 volume of $3.8 \%$ sodium citrate. Platelet-rich plasma (PRP) was obtained from blood samples by centrifugation at $155 \mathrm{x}$ g for $12 \mathrm{~min}$ at room temperature. Platelet-poor plasma (PPP) was prepared from the residual blood by centrifugation at 2,500 $\mathrm{x} g$ for $15 \mathrm{~min}$. All participants signed an informed consent agreement after receiving a detailed explanation, and the study was approved by the Ethics Committee of Gifu University Graduate School of Medicine, Gifu, Japan.

Measurement of platelet aggregation induced by ADP. Platelet aggregation using citrated PRP was carried out in a PA-200 aggregometer (Kowa Co., Ltd., Tokyo, Japan), which can determine the size of platelet aggregates based upon particle counting using laser scattering methods (small size, 9-25 mm; medium size, $25-50 \mathrm{~mm}$; large size, $50-70 \mathrm{~mm}$ ), at $37^{\circ} \mathrm{C}$ with a stirring speed of $800 \mathrm{rpm}$. The platelets were pre-incubated for $1 \mathrm{~min}$, and then platelet aggregation was monitored for $4 \mathrm{~min}$. The percentage of transmittance of the isolated platelets was recorded as $0 \%$, and that of PPP (blank) was recorded as $100 \%$. When indicated, TPO was administered to PRP 15 min prior to with stimulation ADP (pre-treatment), simultaneously with ADP (simultaneous treatment), and 2 min following stimulation with ADP (post-treatment).

Protein preparation following stimulation with ADP. Following stimulation with ADP, platelet aggregation was terminated by the addition of an ice-cold EDTA (10 mM) solution for $5 \mathrm{~min}$ for the western blot analysis samples or for $30 \mathrm{~min}$ for enzymelinked immunosorbent assay (ELISA) samples. The mixture was centrifuged at $10,000 \times \mathrm{g}$ at $4^{\circ} \mathrm{C}$ for $2 \mathrm{~min}$. The supernatant was isolated and stored at $-30^{\circ} \mathrm{C}$ for subsequent ELISA to measure the levels of PDGF-AB and sCD40L. For western blot analysis, the pellet was washed twice with PBS and then lysed and immediately boiled in lysis buffer containing $62.5 \mathrm{mM}$ Tris/Cl, pH 6.8, 2\% sodium dodecyl sulfate (SDS), $50 \mathrm{mM}$ dithiothreitol and $10 \%$ glycerol.

Western blot analysis. Western blot analysis was performed as previously described (8). Briefly, SDS-polyacrolamide gel electrophoresis (PAGE) was performed according to the method described in the study by Laemmli (19) in a $10 \%$ polyacrylamide gel. Proteins in the gel were transferred onto polyvinylidine fluoride (PVDF) membranes. The membranes were blocked with $5 \%$ fat-free dry milk in Tris-buffered saline with $0.1 \%$ Tween-20 (TBS-T, 20 mM Tris- $\mathrm{HCl}$; pH 7.6, $137 \mathrm{mM} \mathrm{NaCl}$, $0.1 \%$ Tween-20) for $1 \mathrm{~h}$ prior to incubation with the indicated primary antibodies overnight. The primary antibodies used in the study were against GAPDH, HSP27, phospho-HSP27 (Ser-15), phospho-HSP27 (Ser-78), phospho-HSP27 (Ser-82), p38 MAP kinase and phospho-p38 MAP kinase antibodies. Peroxidase-labeled anti-goat IgG or anti-rabbit IgG antibodies were used as secondary antibodies. The primary and secondary antibodies were diluted by optimum concentration of $5 \%$ fat-free dry milk in TBS-T. Peroxidase activity on PVDF membranes was visualized on X-ray films by means of an ECL western blotting detection system as per the manufacturer's instructions.

Measurement of PDGF-AB and $s C D 40 L$ levels. The PDGF-AB and SCD40L levels in the samples were determined using PDGF-AB Quantikine and sCD40-Ligand Quantikine ELIsySA kits (R\&D Systems, Inc.), respectively, according to the manufacturer's instructions.

Statistical analysis. Unless otherwise stated, representative results from five independent experiments are shown in the figures. The data are presented as the means \pm SEM. The data were analyzed using the Student's t-test, and a p-value $<0.05$ was considered to indicate a statistically significant difference.

\section{Results}

Effect of TPO on ADP-induced platelet aggregation. We first examined the effect of pre-treatment with TPO on ADP-induced platelet aggregation. TPO, which on its own did not induce platelet aggregation, significantly enhanced the platelet aggregation induced by $1 \mathrm{mM}$ ADP (Fig. 1). According to the analysis of the size of the aggregates, the percentage of large-size aggregates $(50-70 \mathrm{~mm})$ was significantly increased from 34 to $57 \%$. On the other hand, TPO markedly decreased the number of small aggregates. 


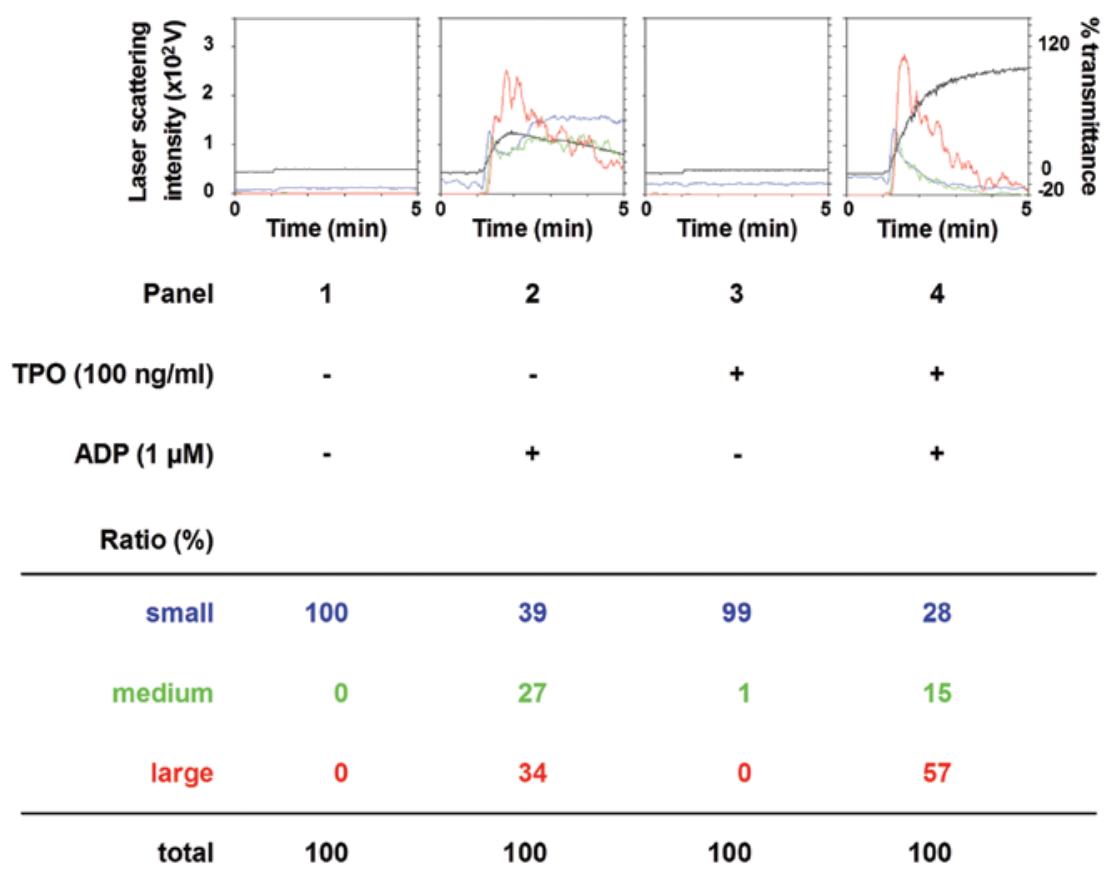

Figure 1. Effects of pre-treatment with thrombopoietin (TPO) on adenosine diphosphate (ADP)-induced platelet aggregation. Platelet-rich plasma (PRP) was pre-treated with $100 \mathrm{ng} / \mathrm{ml} \mathrm{TPO}$ for $15 \mathrm{~min}$, followed by stimulation with $1 \mathrm{mM}$ ADP for $4 \mathrm{~min}$. To terminate the reaction, ice-cold EDTA (10 mM) solution was added. The black line indicates the percentage of transmittance of each sample [the isolated platelets were recorded as 0\%, and platelet-poor plasma (PPP) was recorded as $100 \%]$. The blue line indicates small aggregates $(9-25 \mathrm{~mm})$; green line indicates medium aggregates $(25-50 \mathrm{~mm})$; and the red line indicates large aggregates $(50-70 \mathrm{~mm})$. The distributions $(\%)$ of aggregated particle size were measured using the laser scattering method. Representative results from five independent experiments are shown.

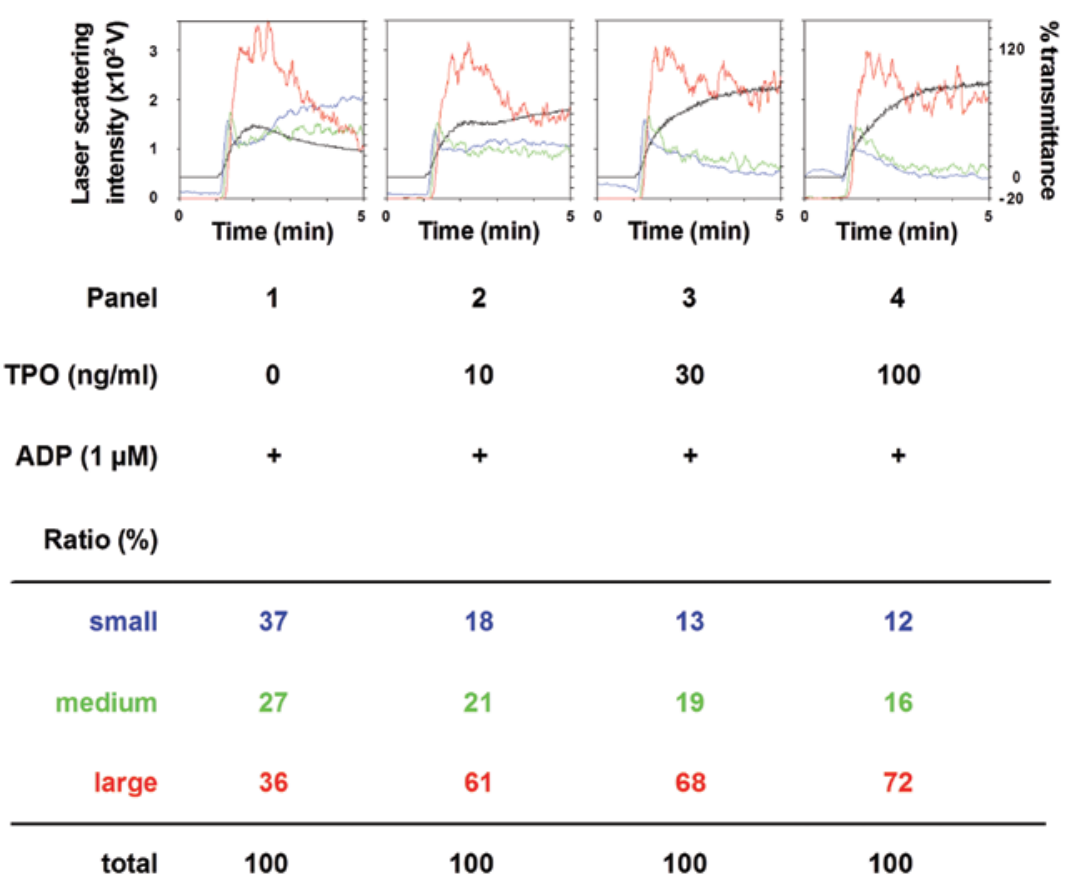

Figure 2. Dose-dependent effect of pre-treatment with thrombopoietin (TPO) on adenosine diphosphate (ADP)-induced platelet aggregation. Platelet-rich plasma (PRP) was pre-treated with various doses of TPO for $15 \mathrm{~min}$, followed by stimulation with $1 \mathrm{mM}$ ADP for 4 min. To terminate the reaction, ice-cold EDTA (10 $\mathrm{mM}$ ) solution was added. The black line indicates the percentage of transmittance of each samples [the isolated platelets was recorded as 0\%, and platelet-poor plasma (PPP) was recorded as $100 \%]$. The blue line indicates small aggregates $(9-25 \mathrm{~mm})$; green line indicates medium aggregates $(25-50 \mathrm{~mm})$; and the red line indicates large aggregates $(50-70 \mathrm{~mm})$. The distributions $(\%)$ of aggregated particle size were measured using the laser scattering method. Representative results from five independent experiments are shown.

We then examined the dose-dependent effect of TPO on the ADP (1 mM)-induced platelet aggregation. The amplifying effect of TPO (between 10 and $100 \mathrm{mM}$ ) was dose-dependent
(Fig. 2). The number of large aggregates was dose-dependently increased by TPO, whereas the number of small aggregates was decreased. 


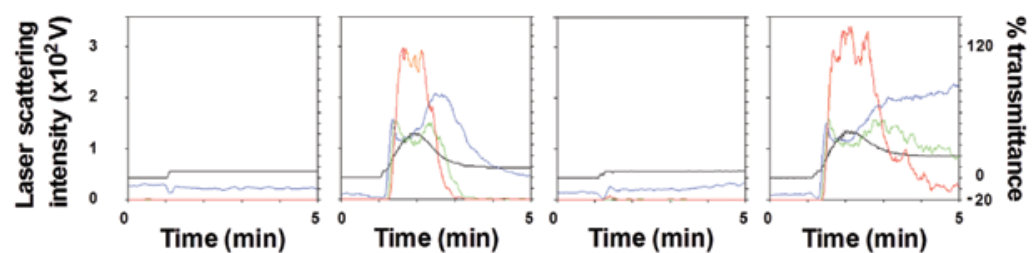

Pane

2

3

4

TPO (100 ng/ml)

$\operatorname{ADP}(1 \mu \mathrm{M})$

Ratio (\%)

\begin{tabular}{rrrcc}
\hline small & 100 & 47 & 96 & 41 \\
medium & 0 & 21 & 2 & 27 \\
large & 0 & 32 & 2 & 32 \\
\hline total & 100 & 100 & 100 & 100
\end{tabular}

Figure 3. Effect of a combination of thrombopoietin (TPO) and adenosine diphosphate (ADP) on platelet aggregation. Platelet-rich plasma (PRP) was stimulated simultaneously with $100 \mathrm{ng} / \mathrm{ml}$ TPO and $1 \mathrm{mM}$ ADP for $4 \mathrm{~min}$. The reaction was terminated by the addition of ice-cold EDTA (10 mM) solution. The black line indicates the percentage of transmittance of each samples [the isolated platelets was recorded as $0 \%$, and platelet-poor plasma (PPP) was recorded as $100 \%$ ]. The blue line indicates small aggregates $(9-25 \mathrm{~mm})$; green line indicates medium aggregates $(25-50 \mathrm{~mm})$; and the red line indicates large aggregates $(50-70 \mathrm{~mm})$. The distributions $(\%)$ of aggregated particle size were measured using the laser scattering method. Representative results from five independent experiments are shown.
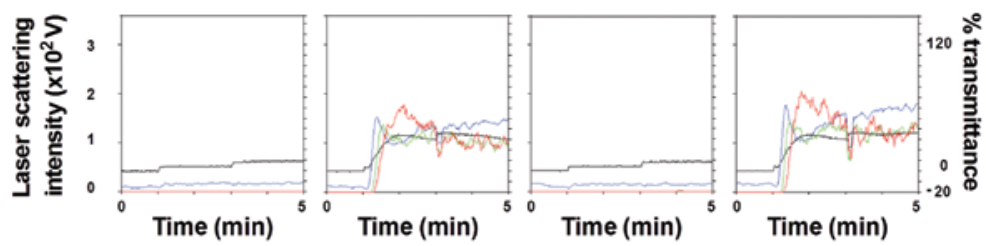

Panel

1

2

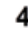

TPO (100 ng/ml)

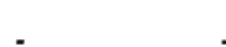

\begin{tabular}{rcccc} 
TPO $(100 \mathrm{ng} / \mathrm{ml})$ & - & - & + & + \\
$\operatorname{ADP}(1 \mu \mathrm{M})$ & - & + & - & + \\
Ratio $(\%)$ & & & & \\
\hline small & 100 & 38 & 99 & 37 \\
medium & 0 & 30 & 1 & 30 \\
large & 0 & 32 & 0 & 33 \\
\hline total & 100 & 100 & 100 & 100
\end{tabular}

Figure 4. Effect of post-treatment with thrombopoietin (TPO) on adenosine diphosphate (ADP)-induced platelet aggregation. Platelet-rich plasma (PRP) was stimulated with $1 \mathrm{mM}$ ADP, and $100 \mathrm{ng} / \mathrm{ml}$ TPO was administered after $2 \mathrm{~min}$. After $2 \mathrm{~min}$, the reaction was terminated by the addition of ice-cold EDTA $(10 \mathrm{mM})$ solution. The black line indicates the percentage of transmittance of each samples [the isolated platelets was recorded as $0 \%$, and platelet-poor plasma (PPP) was recorded as $100 \%$ ]. The blue line indicates small aggregates $(9-25 \mathrm{~mm})$; green line indicates medium aggregates (25-50 mm); and the red line indicates large aggregates $(50-70 \mathrm{~mm})$. The distributions $(\%)$ of aggregated particle size were measured using the laser scattering method. Representative results from five independent experiments are shown.

In order to clarify the exact mechanism of action of TPO in ADP-induced platelet activation, we examined the effect of a combination of TPO and ADP on platelet aggregation. In contrast to pre-treatment with TPO, the simultaneous stimulation with TPO and ADP did not further enhance platelet aggregation compared to treatment with ADP alone (Fig. 3). 
A

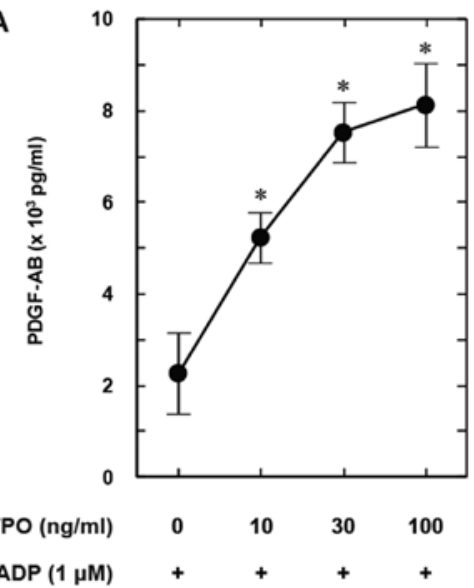

B

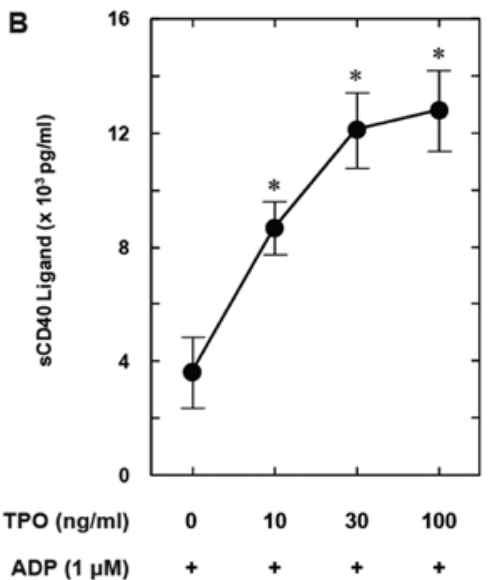

Figure 5. Effects of pre-treatment with thrombopoietin (TPO) on the adenosine diphosphate (ADP)-induced (A) platelet-derived growth factor (PDGF)-AB secretion and (B) the release of soluble CD40 ligand (sCD40L) from human platelets. Platelet-rich plasma (PRP) was pre-treated with various concentrations of TPO for $15 \mathrm{~min}$, followed by stimulation with $1 \mathrm{mM}$ ADP for $30 \mathrm{~min}$. The reaction was terminated by the addition of ice-cold EDTA (10 mM) solution. The mixture was centrifuged at $10,000 \mathrm{x}$ g at $4^{\circ} \mathrm{C}$ and the supernatants were then subjected to enzyme-linked immunosorbent assay (ELISA) for PDGF-AB and $\mathrm{sCD} 40 \mathrm{~L}$. Results from five independent experiments are shown. Each value represents the mean $\pm \mathrm{SEM}$. " $\mathrm{p}<0.05$, compared to the value of ADP alone.
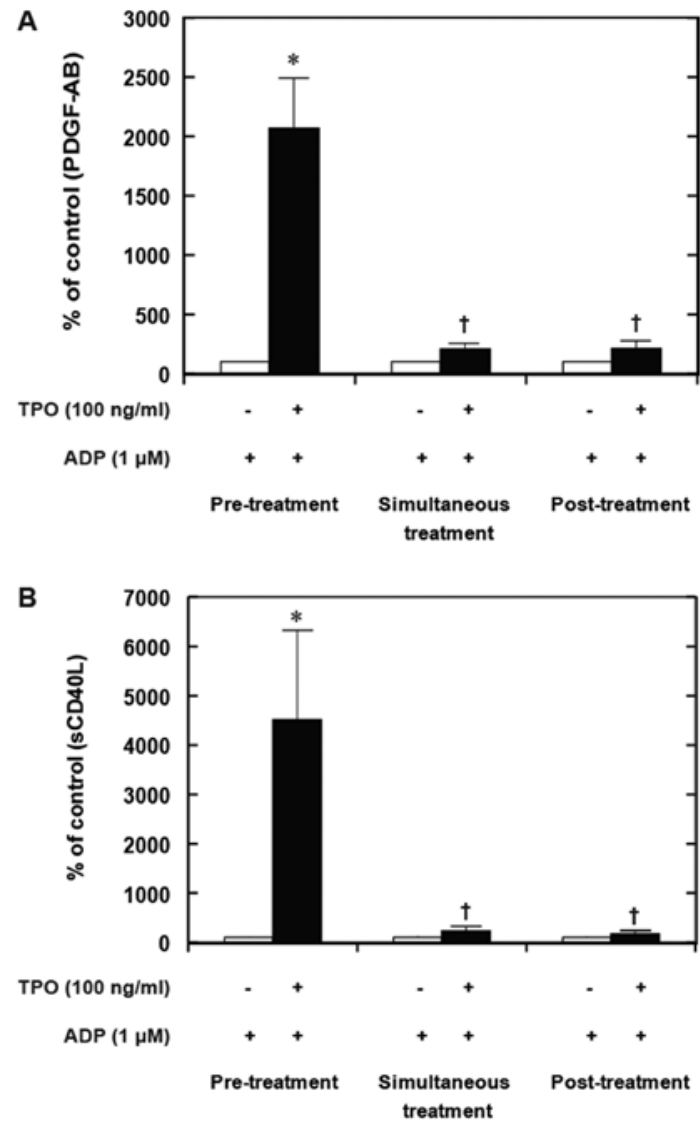

Figure 6. Effects of thrombopoietin (TPO) on the adenosine diphosphate (ADP)-induced (A) platelet-derived growth factor (PDGF)-AB secretion and (B) the release of soluble CD40 ligand (sCD40L) from human platelets. Platelet-rich plasma (PRP) was stimulated simultaneously by $100 \mathrm{ng} / \mathrm{ml}$ TPO and $1 \mathrm{mM}$ ADP (simultaneous treatment) or stimulated with $1 \mathrm{mM} \mathrm{ADP}$, and $100 \mathrm{ng} / \mathrm{ml}$ TPO was administered after $2 \mathrm{~min}$ (post-treatment). The reaction was terminated by the addition of ice-cold EDTA $(10 \mathrm{mM})$ solution after 30 min following stimulation with ADP. The mixture was centrifuged at $10,000 \mathrm{xg}$ at $4^{\circ} \mathrm{C}$ and the supernatants were then subjected to enzyme-linked immunosorbent assay (ELISA) for PDGF-AB and SCD40L. Values were expressed as a percentage (\%) of ADP $1 \mathrm{mM}$ alone. Results from five independent experiments are shown. Each value represents the mean \pm SEM. ${ }^{*} p<0.05$, compared to the value of ADP alone, ${ }^{\dagger} \mathrm{p}<0.05$, compared to the value of ADP and pre-treatment with TPO.
In addition, post-treatment with TPO following stimulation with ADP had no additional effect on the platelet aggregation induced by ADP alone (Fig. 4).

Effects of TPO on ADP-induced PDGF-AB secretion and release of $s C D 4 O L$. Pre-treatment with TPO (between 10 and $100 \mathrm{ng} / \mathrm{ml}$ ) significantly enhanced the ADP-induced PDGF-AB secretion in a dose-dependent manner (Fig. 5A). In addition, the release of sCD40L stimulated by ADP was dose-dependently amplified following pre-treatment with TPO (Fig. 5B).

On the other hand, the simultaneous stimulation with TPO and ADP did not further enhance the PDGF-AB secretion compared to treatment with ADP alone (Fig. 6A). Additionally, post-treatment with TPO following stimulation with ADP failed to amplify the ADP-induced release of sCD40L (Fig. 6B).

Effects of TPO on the ADP-induced phosphorylation of p38 MAP kinase and HSP27 in human platelets. We have previously shown that ADP induces HSP27 phosphorylation via the activation of the p38 MAP kinase in human platelets, resulting in the stimulation of PDGF-AB secretion and the release of $\mathrm{sCD} 40 \mathrm{~L}$ (8). Therefore, we examined the effect of pre-treatment with TPO on the ADP-induced phosphorylation of p38 MAP kinase and HSP27. Pre-treatment with TPO (between 10 and $100 \mathrm{mM}$ ), which on its own had little effect on p38 MAP kinase phosphorylation, dose-dependently enhanced the ADP-induced phosphorylation of p38 MAP kinase in a dose-dependent manner (Fig. 7A). However, as regards the simultaneous stimulation with TPO and ADP or post-treatment with TPO, the phosphorylated levels of p38 MAP kinase were similar to those induced by treatment with ADP alone (Fig. 7B and C).

Pre-treatment with TPO, which alone had little effect on the HSP27 phosphorylation (data not shown), markedly enhanced the ADP-induced phosphorylation of HSP27 at three serine residues (Ser-15, Ser-78 and Ser-82) (Fig. 8). The amplifying effects of TPO (between 10 and $100 \mathrm{mM}$ ) were dose-dependent. On the contrary, simultaneous treatment 
A

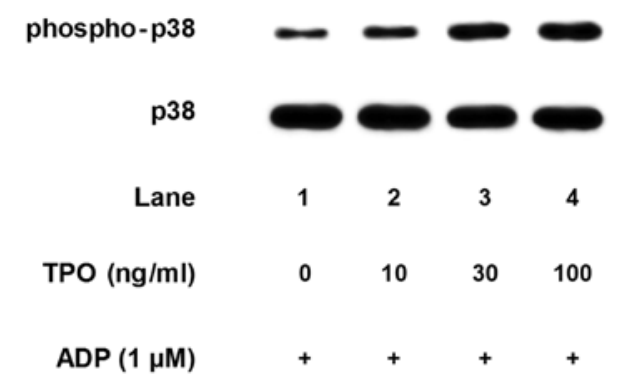

B

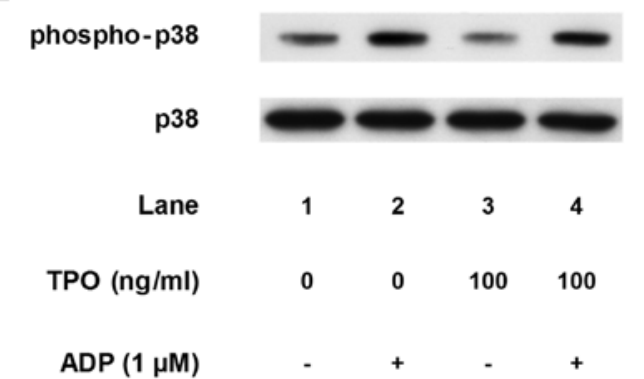

C

phospho-p38

p38

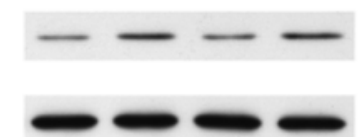

Lane

TPO (ng/ml)

$\operatorname{ADP}(1 \mu \mathrm{M})$
phospho-HSP27 (Ser-15)

phospho-HSP27 (Ser-78)

phospho-HSP27 (Ser-82)

HSP27

GAPDH

Lane

TPO (ng/ml)

ADP $(1 \mu \mathrm{M})$
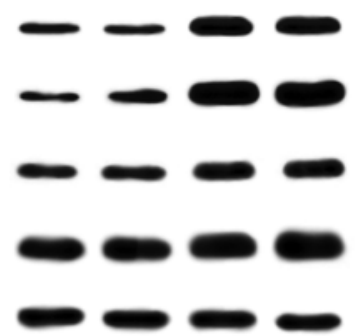

$\begin{array}{rrrr}1 & 2 & 3 & 4 \\ 0 & 10 & 30 & 100\end{array}$

$+\quad+\quad+$

Figure 8. Effect of pre-treatment with thrombopoietin (TPO) on the adenosine diphosphate (ADP)-induced phosphorylation of phosphorylation of heat shock protein 27 (HSP27) in human platelets. Platelet-rich plasma (PRP) was pre-treated with various doses of TPO for $15 \mathrm{~min}$, followed by stimulation with $1 \mathrm{mM}$ ADP for $4 \mathrm{~min}$. The reaction was terminated by the addition of ice-cold EDTA $(10 \mathrm{mM})$ solution. The protein extracts were subjected to SDS-PAGE using antibodies against phospho-specific HSP27 (Ser-15, Ser-78 and Ser-82), HSP27 and GAPDH. Representative results from three independent experiments are shown.

human platelet activation. Based on these results, it is possible that TPO plays a preconditioning role, and acts synergistically with ADP in human platelet activation.

We have previously demonstrated that ADP stimulates the phosphorylation of HSP27 via the activation of p38 MAP kinase in human platelets and that the ADP-induced phosphorylation of HSP27 via the p38 MAP kinase pathway correlates with PDGF-AB secretion and the release of $\mathrm{sCD} 40 \mathrm{~L}$ from human platelets (8). Thus, in this study, we examined the effect of TPO administration on the phosphorylation of p38 MAP kinase and HSP27. It has been reported that TPO amplifies the ADP-stimulated activation of p38 MAP kinase in platelets (14). We found that pre-treatment with TPO markedly enhanced the ADP-induced phosphorylation levels of HSP27 at three serine residues (Ser-15, Ser-78 and Ser-82) in addition to p38 MAP kinase in human platelets. However, the simultaneous stimulation with TPO and ADP or post-treatment with TPO failed to affect the ADP-induced phosphorylation of p38 MAP kinase and HSP27. It appears that the PDGF-AB secretion and release of $\mathrm{SCD} 40 \mathrm{~L}$ enhanced by TPO correlate with the enhanced phosphorylation of p38 MAP kinase and HSP27. Based on these findings, it is possible that amplification of the ADP-induced platelet activation by pre-treatment with TPO is at least in part, due to the upregulation of HSP27 phosphorylation via the p38 MAP kinase pathway.

It is well recognized that activated platelets result in degranulation, such as PDGF-AB secretion and the release of a variety of agents, such as SCD40L. It is well known that PDGF-AB is a potent growth factor which induces the proliferation of vascular smooth muscle cells and plays a crucial role in the development of atherosclerosis. On the other hand, the release of sCD40L from platelets activates CD40 in vascular endothelial cells and smooth muscle cells, and induces a variety of pro-inflammatory and pro-atherogenic responses (21). Elevated sCD40L levels have been observed in patients with acute coronary syndrome (22).

sCD40L has been reported to stimulate the release of inflam-

(1 mM). Therefore, our findings suggest the importance of pre-treatment with TPO in the enhancement of ADP-induced 
matory substances from dense granules in human platelets (23). In the present study, we demonstrated that TPO enhanced the PDGF-AB secretion and the release of sCD40L from platelets activated by ADP. Thus, it is possible that TPO acts as a proinflammatory mediator, resulting in inflammation. It has been shown that serum TPO levels in patients with serious diseases, such as sepsis, trauma and thrombocytopenia are higher than normal (24-26). It has also been shown that in patients with sepsis, serum TPO levels are closely associated with the severity of the disease $(25,26)$. Taking these findings into account, it is possible that inflammatory agents, whose release is increased from activated platelets by TPO in patients with these disorders, may aggravate the pathological conditions. Thus, the blockade of TPO-amplifying effects on platelet function seems to be crucial in preventing the acceleration of pathological states. Further studies are required to clarify the precise mechanism of action of TPO in platelet activation.

In conclusion, the results from the present study strongly suggest that pre-treatment with TPO amplifies ADP-induced HSP27 phosphorylation via the p38 MAP kinase pathway in human platelets.

\section{Acknowledgements}

We are very grateful to Yumiko Kurokawa for her skillful technical assistance. This study was supported in part by Grants-in-Aid for Scientific Research (nos. 20590565 and 20591825) from the Ministry of Education, Science, Sports and Culture of Japan and Research Grants for Longevity Sciences (22A-4) and Research on Proteomics from the Ministry of Health, Labour and Welfare of Japan.

\section{References}

1. Kahner BN, Shankar H, Murugappan S, Prasad GL and Kunapuli SP: Nucleotide receptor signaling in platelets. J Thromb Haemost 4: 2317-2326, 2006.

2. Li Z, Delaney MK, O'Brien KA and Du X: Signaling during platelet adhesion and activation. Arterioscler Thromb Vasc Biol 30: 2341-2349, 2010.

3. Dangelmaier C, Jin J, Daniel JL, Smith JB and Kunapuli SP: The P2Y1 receptor mediates ADP-induced p38 kinase-activating factor generation in human platelets. Eur J Biochem 267: 2283-2289, 2000.

4. Falker K, Lange D and Presek P: ADP secretion and subsequent P2Y12 receptor signalling play a crucial role in thrombin-induced ERK2 activation in human platelets. Thromb Haemost 92: 114-123, 2004

5. Stegner D and Nieswandt B: Platelet receptor signaling in thrombus formation. J Mol Med (Berl) 89: 109-121, 2011.

6. Mymrikov EV, Seit-Nebi AS and Gusev NB: Large potentials of small heat shock proteins. Physiol Rev 91: 1123-1159, 2011.

7. Zhu Y, O'Neill S, Saklatvala J, Tassi L and Mendelsohn ME: Phosphorylated HSP27 associates with the activation-dependent cytoskeleton in human platelets. Blood 84: 3715-3723, 1994.

8. Kato H, Takai S, Matsushima-Nishiwaki R, Adachi S, Minamitani C, Otsuka T, Tokuda H, Akamatsu S, Doi T, Ogura S and Kozawa O: HSP27 phosphorylation is correlated with ADP-induced platelet granule secretion. Arch Biochem Biophys 475: 80-86, 2008.
9. Ellis MH, Avraham H and Groopman JE: The regulation of megakaryocytopoiesis. Blood Rev 9: 1-6, 1995.

10. Ezumi Y, Takayama $\mathrm{H}$ and Okuma M: Thrombopoietin, c-Mpl ligand, induces tyrosine phosphorylation of Tyk2, JAK2, and STAT3, and enhances agonists-induced aggregation in platelets in vitro. FEBS Lett 374: 48-52, 1995.

11. Kojima H, Hamazaki Y, Nagata Y, Todokoro K, Nagasawa T and Abe T: Modulation of platelet activation in vitro by thrombopoietin. Thromb Haemost 74: 1541-1545, 1995.

12. Rodriguez-Linares B and Watson SP: Thrombopoietin potentiates activation of human platelets in association with JAK2 and TYK2 phosphorylation. Biochem J 316: 93-98, 1996.

13. Kubota Y, Arai T, Tanaka T, Yamaoka G, Kiuchi H, Kajikawa T, Kawanishi K, Ohnishi H, Yamachi M, Takahara J and Iriono S: Thrombopoietin modulates platelet activation in vitro through protein-tyrosine phosphorylation. Stem Cells 14: 439-444, 1996.

14. Ezumi Y, Nishida E, Uchiyama $\mathrm{T}$ and Takayama $\mathrm{H}$ : Thrombopoietin potentiates agonist-stimulated activation of p38 mitogen-activated protein kinase in human platelets. Biochem Biophys Res Commun 261: 58-63, 1999.

15. Kojima H, Shinagawa A, Shimizu S, Kanada H, Hibi M, Hirano T and Nagasawa T: Role of phosphatidylinositol-3 kinase and its association with Gab1 in thrombopoietin-mediated up-regulation of platelet function. Exp Hematol 29: 616-622, 2001.

16. Chen J, Herceg-Harjacek L, Groopman JE and Grabarek J: Regulation of platelet activation in vitro by the c-Mpl ligand, thrombopoietin. Blood 86: 4054-4062, 1995.

17. Oda A, Miyakawa Y, Druker BJ, Ozaki K, Yabusaki K, Shirasawa Y, Handa M, Kato T, Miyazaki H, Shimosaka A and Ikeda Y: Thrombopoietin primes human platelet aggregation induced by shear stress and by multiple agonists. Blood 87: 4664-4670, 1996.

18. Eilers M, Schulze H, Welte K and Ballmaier M: Thrombopoietin acts synergistically on $\mathrm{Ca}(2+)$ mobilization in platelets caused by ADP or thrombin receptor agonist peptide. Biochem Biophys Res Commun 263: 230-238, 1999.

19. Laemmli UK: Cleavage of structural proteins during the assembly of the head of bacteriophage T4. Nature 227: 680-685, 1970.

20. Wun T, Paglieroni T, Hammond WP, Kaushansky $\mathrm{K}$ and Foster DC: Thrombopoietin is synergistic with other hematopoietic growth factors and physiologic platelet agonists for platelet activation in vitro. Am J Hematol 54: 225-232, 1997.

21. Henn V, Slupsky JR, Grafe M, Anagnostopoulos I, Forster R, Muller-Berghaus G and Kroczek RA: D40 ligand on activated platelets triggers an inflammatory reaction of endothelial cells. Nature 391: 591-594, 1998.

22. Heeschen C, Dimmeler S, Hamm CW, van den Brand MJ, Boersma E, Zeiher AM and Simooms ML: Soluble CD40 ligand in acute coronary syndromes. N Engl J Med 348: 1104-1111, 2003.

23. Inwald DP, McDowall A, Peters MJ, Callard RE and Klein NJ: CD40 is constitutively expressed on platelets and provides a novel mechanism for platelet activation. Circ Res 92: 1041-1048, 2003.

24. Kaushansky K: Thrombopoietin. N Engl J Med 339: 746-754, 1998.

25. Zakynthinos SG, Papanikolaou S, Theodoridis T, Zakynthinos EG, Christopoulou-Kokkinou V, Katsaris G and Mavrommatis AC: Sepsis severity is the major determinant of circulating thrombopoietin levels in septic patients. Crit Care Med 32: 1004-1010, 2004.

26. Lupia E, Bosco O, Mariano F, Dondi AE, Goffi A, Spatola T, Cuccurullo A, Tizzani P, Brondino G, Stella M and Montrucchio G: Elevated thrombopoietin in plasma of burned patients without and with sepsis enhances platelet activation. J Thromb Haemost 7: 1000-1008, 2009. 